\title{
Spacelike deformations: higher-helicity fields from scalar fields
}

\author{
Vincenzo Morinelli ${ }^{1}$ (D) $\cdot$ Karl-Henning Rehren ${ }^{2}$ (D)
}

Received: 25 May 2019 / Revised: 11 March 2020 / Accepted: 28 May 2020 / Published online: 11 June 2020

(c) The Author(s) 2020

\begin{abstract}
In contrast to Hamiltonian perturbation theory which changes the time evolution, "spacelike deformations" proceed by changing the translations (momentum operators). The free Maxwell theory is only the first member of an infinite family of spacelike deformations of the complex massless Klein-Gordon quantum field into fields of higher helicity. A similar but simpler instance of spacelike deformation allows to increase the mass of scalar fields.
\end{abstract}

Keywords Quantum field theory · Representation theory · Deformation theory · Higher helicity · Operator algebra

Mathematics Subject Classification 81R05 · 81R15 · 22E43

\section{Introduction}

The basic idea of Hamiltonian perturbation theory is to start from a time zero algebra ("canonical commutation relations") equipped with a free time evolution, and perturb the free Hamiltonian such that the observables at later time $\Phi(t):=e^{i H t} \Phi_{0} e^{-i H t}$ (where $H$ is the perturbed Hamiltonian) deviate from the free ones. We present here

\footnotetext{
Vincenzo Morinelli: Titolare di un Assegno di Ricerca dell' Istituto Nazionale di Alta Matematica (INdAM fellowship).

$凶$ Karl-Henning Rehren

rehren@theorie.physik.uni-goettingen.de

Vincenzo Morinelli morinell@mat.uniroma2.it

1 Dipartimento di Matematica, Università di Roma Tor Vergata, Via della Ricerca Scientifica, 1, 00133 Rome, Italy

2 Institut für Theoretische Physik, Universität Göttingen, 37077 Göttingen, Germany
} 
a "complementary" deformation scheme for free quantum field theories: fixing the algebra along the time axis, we deform the space translations, so as to obtain a different local quantum field theory in Minkowski space.

Despite the apparent similarity, there are many differences, though. Hamiltonian Perturbation Theory (PT) is well-known to be obstructed by Haag's theorem, which implies that the perturbation is possible on the same Hilbert space only locally. Globally, the perturbed vacuum state is not a state in the "free Hilbert space", so that one is forced to change the representation of the time zero algebra. The need of renormalization of the mass also shows that one is even forced to change the time zero algebra itself. More precisely, interacting quantum fields in general do not even exist as distributions at a fixed time (see, e.g., [14,15]).

A recent approach [3], designed to avoid these obstructions, uses instead of a CCR time-zero algebra, an abstract "off-shell" $\mathrm{C}^{*}$-algebra of kinematical fields on spacetime which supports a large class of dynamics (one-parameter groups of time-evolution automorphisms). The invariant states under each dynamics, however, annihilate different ideals of the algebra ("field equations"), such that the corresponding GNS Hilbert spaces cannot be identified for any time-zero subalgebra.

In contrast, Wightman quantum fields can always be restricted to the time axis [2]. Our spacelike deformations are globally well-defined on a subspace of the original Hilbert space. They consist in a redefinition of the generators of the spacelike translations (momentum operators). The perturbed fields away from the time axis are then defined as $\Phi(t, \mathbf{x})=e^{i x^{k} \widetilde{P}_{k}} \Phi_{0}(t, 0) e^{-i x^{k} \widetilde{P}_{k}}$, where $\widetilde{P}_{k}$ are the deformed generators.

In Hamiltonian PT, the "field content" is fixed by the choice of the free theory. The "particle content" is determined by the spectrum of the (renormalized) perturbed Hamiltonian, and may well change, e.g., when the interacting theory has bound states, or confinement occurs. Yet, the relation to the free particle content is usually not entirely lost.

In contrast, our spacelike deformations are (non-perturbative) algebraic deformations that drastically change the field content without changing the Hamiltonian: e.g., one obtains the free Maxwell field by a deformation of a massless free scalar field.

In fact, we know spacelike deformations only for free fields, producing massless higher helicity fields from scalar ones (Sect. 2.3), or massive scalar fields from massless ones (Sect. 3). The reason is that (a) we work on the one-particle space, from which the deformation passes to the Fock space by "second quantization"; and (b) the construction is essentially representation-theoretic. Namely, it transfers the representation of the Poincaré group of one theory to a subspace of the representation space of the other theory by a unitary operator, intertwining the subgroup that fixes the time-axis. ${ }^{1}$ We present the deformed generators as (nonlinear) functions of the undeformed generators.

\footnotetext{
1 This subgroup consists of the rotations and the Möbius group Möb. The latter is familiar from chiral conformal QFT on the light-ray of two-dimensional CFT. Here, Möb acts in the same way by fractional linear transformations on the (compactified) time axis. It is generated by the time translations and the "conformal inversion" $x=(t, \mathbf{x}) \mapsto \frac{(-t, \mathbf{x})}{t^{2}-\mathbf{x}^{2}}$, which becomes $t \mapsto-1 / t$ on the time axis.
} 
We are therefore far from "interactions via deformation"; but our models illustrate the potential of a new approach, and more sophisticated new ideas may emerge from the present simple prototypes.

\section{Helicity deformations}

\subsection{Background}

The first examples to be demonstrated rely on a recent observation in [9]: For the massless free fields of any integer helicity $h>0$, the one-particle spaces $\mathcal{H}^{(h)}$ are proper subspaces of the one-particle space $\mathcal{H}=\mathcal{H}^{(0)}$ of the complex massless free scalar field. More precisely, $\mathcal{H}^{(h)}$ as representations $U_{h} \oplus U_{-h}$ of the Poincaré group extend to representations $U_{(h)}$ of the conformal group, whose restriction to the subgroup $\mathrm{Möb} \times \mathrm{SO}(3)$ is given by

$$
\left.\mathcal{H}^{(h)}\right|_{\mathrm{Möb} \times \mathrm{SO}(3)}=\bigoplus_{\ell=h}^{\infty}\left(U^{(\ell+1)} \otimes \mathcal{D}^{(\ell)}\right) \oplus\left(U^{(\ell+1)} \otimes \mathcal{D}^{(\ell)}\right),
$$

where $\mathcal{D}^{(\ell)}$ are the spin- $\ell$ representations of $\mathrm{SO}(3)$, and $U^{(d)}$ are the irreducible positive-energy representations of Möb with lowest eigenvalue $d$ of the "conformal Hamiltonian" $L_{0}=\frac{1}{2}\left(P_{0}+K_{0}\right)$. (We follow rather standard notation for the generators of the conformal group, fixing conventions in Sect. 2.2; for more details on the relations between the various groups and representations, see Sect. 2.2 and [9].) The doubling is due to the "electric" and "magnetic" degrees of freedom. The same decomposition with $h=0$ holds for the complex scalar field, where the doubling corresponds to the subspaces of charge \pm 1 .

$\mathrm{Möb} \times \mathrm{SO}(3)$ is the subgroup of the conformal group that fixes the time axis $\mathbf{x}=0$. The vectors transforming in the displayed subrepresentations are spacelike derivatives of fields on the time axis, that transform like quasiprimary fields under Möb, applied to the vacuum vector $\Omega$. For the scalar field, these fields are simply [4]

$$
\left.Y_{\ell}(\nabla) \varphi^{(*)}(x)\right|_{x=(t, \mathbf{0})}
$$

$\left(\varphi^{(*)}\right.$ stands for both $\varphi$ and the conjugate field $\left.\varphi^{*}\right)$ with harmonic ${ }^{2}$ homogeneous polynomials $Y_{\ell}$ of degree $\ell$, transforming like spin- $\ell$ multiplets of quasiprimary fields of scaling dimension $d=\ell+1$. For $h>0$, when the electric and magnetic fields are combined into a complex field tensor $F_{j_{1} \ldots j_{h}}=E_{j_{1} \ldots j_{h}}+i B_{j_{1} \ldots j_{h}}$, the equations of motion impose linear relations among the fields $\left.\nabla_{i_{1}} \ldots \nabla_{i_{r}} F_{j_{1} \ldots j_{h}}(x)\right|_{x=(t, \mathbf{0})}$. The decomposition (2.1) implies that the time-axis field content is given by exactly two quasiprimary spin- $\ell$ multiplets (one for both $F$ and $F^{*}$ ) of scaling dimension $d=\ell+1$ for each $\ell \geq h$, and that the Casimir operator of the Lie algebra of the rotations (eigenvalues $\ell(\ell+1)$ ) and the Casimir operator of the Lie algebra of the Möbius group (eigenvalues $d(d-1)$ ) coincide in the one-particle representation.

\footnotetext{
${ }^{2}$ A polynomial in $\mathbb{R}^{n}$ is harmonic iff $\Delta Y=0$ where $\Delta=\sum_{k=1}^{n} \partial_{k}^{2}$ is the Laplacian. In this paper, $n=3$.
} 
In this count, as $h$ increases, the field content decreases. The lowest fields of the scalar theory are given by $\varphi^{(*)}(t, \mathbf{0})(\ell=0, d=1)$ and $\nabla \varphi^{(*)}(t, \mathbf{0})(\ell=1, d=2)$, while, e.g., the Maxwell theory starts at $\ell=1, d=2$ with the vector field $\mathbf{F}^{(*)}=$ $\mathbf{E} \pm i \mathbf{B}$. In this sense, contrary to intuition, the higher-helicity theories have less degrees of freedom than the lower- $h$ theories.

In [9], these facts were exploited to estimate the trace of $e^{-\beta L_{0}}$, whose finiteness then implies the split property for all finite-helicity massles free quantum field theories. Here, we take them as the starting point of spacelike deformation, as already speculated in [9]: we unitarily identify the common subrepresentations $\ell \geq h$ of the subgroup Möb $\times \mathrm{SO}(3)$, and find the necessary modification of the representation of the remaining generators.

To illustrate the idea, consider the case $h=1$ (Maxwell). The Maxwell equations for $\mathbf{F}$ read

$$
\nabla \cdot \mathbf{F}=0, \quad \nabla \times \mathbf{F}=i \partial_{t} \mathbf{F}
$$

The component fields $F_{k}(t, \mathbf{0})$ on the time axis transform in the same way under Möbius transformations of the time axis and rotations, like the fields $\nabla_{k} \varphi(x, \mathbf{0})$ of the complex massless Klein-Gordon theory. Similarly, the fields $\left(\nabla_{i} F_{j}+\nabla_{j} F_{i}\right)(t, \mathbf{0})$ of spin 2 transform in the same way as the fields $\left(\nabla_{i} \nabla_{j}-\frac{1}{3} \delta_{i j} \Delta\right) \varphi(t, \mathbf{0})$.

Because the representations of the Möbius and rotation groups on the one-particle spaces are the same-except for the absence of the subrepresentation with $\ell=0$ in the Maxwell theory — we can algebraically identify these pairs of fields along the time axis. We get

$$
\begin{gathered}
F_{i}(t, \mathbf{0}) \stackrel{!}{=} 2 \nabla_{i} \varphi(t, \mathbf{0}) \\
\nabla_{i} F_{j}(t, \mathbf{0}) \stackrel{!}{=} \alpha \cdot\left(\nabla_{i} \nabla_{j}-\frac{1}{3} \delta_{i j} \Delta\right) \varphi(t, \mathbf{0})+i \varepsilon_{i j k} \partial_{t} \nabla_{k} \varphi(t, \mathbf{0}),
\end{gathered}
$$

where the Maxwell equations dictate the anti-symmetric part in (2.3) as well as the absence of an $\ell=0$ contribution; the two-point function fixes the normalizations, giving $|\alpha|^{2}=12$.

The problem is apparent: the left-hand side of (2.3) is the derivative of the left-hand side of (2.2), which is not true for the right-hand sides. The spatial derivatives being implemented by the momentum operators $P_{k}$, we conclude that while the Möbius and rotation generators of both theories (including the Hamiltonian $P_{0}$ ) can be identified, their spatial momentum operators must differ.

We are going to determine the momentum operators $\widetilde{P}_{k}$ of the Maxwell theory as polynomials of the conformal and charge generators of the Klein-Gordon theory (and along with them the boosts and the generators of spatial special conformal transformations). Then, starting from the identification (2.2) as a definition of the Maxwell field on the time axis, and acting with $\widetilde{U}(\mathbf{x})=e^{i x^{k} \widetilde{P}_{k}}$ on $\varphi(t, \mathbf{0})$, one obtains the Maxwell field everywhere in Minkowski space. The same works for any helicity $h>0$. 
As a second instance, we present the spacelike deformation of the massless scalar field into the massive scalar field in Sect. 3.

The mere existence of such deformations should not be too surprising, given that "all Hilbert spaces are the same". The noticeable facts are that the deformations fix parts of the symmetry, and that they can be given on the remaining generators by explicit formulae.

\subsection{Preliminaries about the conformal Lie algebra}

We denote by $P_{\mu}, M_{\mu \nu}, D, K_{\mu}$ the generators of translations, Lorentz transformations, dilations, and special conformal transformations in the conformal Lie algebra $\mathfrak{s o}(2,4)$, respectively. Their commutators are explicitly

$$
\begin{array}{ll}
i\left[P_{\mu}, P_{\nu}\right]=0, \quad i\left[P_{\mu}, M_{\kappa \lambda}\right]=\eta_{\mu \lambda} P_{\kappa}-\eta_{\mu \kappa} P_{\lambda}, & i\left[M_{\kappa \lambda}, M_{\mu \nu}\right]=\eta_{\kappa \mu} M_{\lambda \nu} \pm \cdots \\
i\left[D, P_{\mu}\right]=P_{\mu}, i\left[D, K_{\mu}\right]=-K_{\mu}, & i\left[D, M_{\kappa \lambda}\right]=0 ; \\
i\left[K_{\mu}, K_{\nu}\right]=0, i\left[M_{\kappa \lambda}, K_{\mu}\right]=\eta_{\kappa \mu} K_{\lambda}-\eta_{\lambda \mu} K_{\kappa}, i\left[P_{\mu}, K_{\nu}\right]=-2 \eta_{\mu \nu} D+2 M_{\mu \nu}
\end{array}
$$

In particular, we have the Lie subalgebras möb:

$$
i\left[D, P_{0}\right]=P_{0}, \quad i\left[P_{0}, K_{0}\right]=-2 D, \quad i\left[D, K_{0}\right]=-K_{0},
$$

and $\mathfrak{s o}(3)$ :

$$
i\left[M_{i j}, M_{k l}\right]=\delta_{j k} M_{i l}-\delta_{j l} M_{i k}-\delta_{i k} M_{j l}+\delta_{i l} M_{j k}
$$

Lemma 2.1 The parity reflection $(t, \mathbf{x}) \mapsto(t,-\mathbf{x})$ defines a symmetric space decomposition of the conformal Lie algebra

$$
\mathfrak{s o}(2,4)=\mathfrak{h} \oplus \mathfrak{m}, \quad[\mathfrak{h}, \mathfrak{h}] \subset \mathfrak{h}, \quad[\mathfrak{h}, \mathfrak{m}] \subset \mathfrak{m}, \quad[\mathfrak{m}, \mathfrak{m}] \subset \mathfrak{h},
$$

where $\mathfrak{h}=\mathfrak{m} \ddot{\mathfrak{b}} \oplus \mathfrak{s o}(3)=\operatorname{Span}\left(P_{0}, D, K_{0}, M_{k l}\right)$ and $\mathfrak{m}=\operatorname{Span}\left(P_{k}, M_{0 k}, K_{k}\right)$. The generators of $\mathfrak{m}$ transform like vectors under $\mathfrak{s o}(3)$ :

$$
i\left[M_{k l}, X_{i}\right]=\delta_{l i} X_{k}-\delta_{k i} X_{l},
$$

and $\mathfrak{m o ̈ b}$ acts on $\mathfrak{m}$ as a $\mathfrak{m o ̈ b}-$ module like

$$
\begin{array}{lll}
i\left[P_{0}, P_{k}\right]=0, & i\left[P_{0}, M_{0 k}\right]=-P_{k}, & i\left[P_{0}, K_{k}\right]=2 M_{0 k}, \\
i\left[D, P_{k}\right]=P_{k}, & i\left[D, M_{0 k}\right]=0, & i\left[D, K_{k}\right]=-K_{k}, \\
i\left[K_{0}, P_{k}\right]=2 M_{0 k}, i\left[K_{0}, M_{0 k}\right]=-K_{k}, & i\left[K_{0}, K_{k}\right]=0 .
\end{array}
$$

In particular, $\left(\operatorname{ad}_{P_{0}}\right)^{3}=0$ and $\left(\operatorname{ad}_{K_{0}}\right)^{3}=0$ on $\mathfrak{m}$.

Proof Immediate from (2.4). 
In the sequel, we shall need the action of the generators on suitable vectors in the $h=0$ representation. This representation is realized on the one-particle space $\mathcal{H}$ of the real massless scalar field $\varphi$. Its two-point function

$$
\left(\varphi(x) \Omega, \varphi\left(x^{\prime}\right) \Omega\right)=(2 \pi)^{-3} \int \frac{\mathrm{d}^{3} p}{2 p^{0}} e^{-i p(x-y)} \quad\left(p^{0}=|\mathbf{p}|\right)
$$

is invariant under the infinitesimal conformal transformations ${ }^{3}$

$$
\begin{array}{ll}
i\left[P_{\mu}, \varphi(x)\right]=\partial_{\mu} \varphi(x), & i\left[M_{\mu \nu}, \varphi(x)\right]=\left(x_{\mu} \partial_{v}-x_{v} \partial_{\mu}\right) \varphi(x) \\
i[D, \varphi(x)]=((x \partial)+1) \varphi(x), & i\left[K_{\mu}, \varphi(x)\right]=\left(2 x_{\mu}(x \partial)-x^{2} \partial_{\mu}+2 x_{\mu}\right) \varphi(x)
\end{array}
$$

Because Wightman fields can be restricted to the time axis [2], they only need smearing in the time variable. We may thus introduce, for polynomials $Y(\mathbf{p})$ on momentum space $\mathbb{R}^{3}$, improper one-particle vectors

$$
|Y\rangle_{t}=\left.Y(-i \nabla) \varphi(\mathbf{x}, t)\right|_{\mathbf{x}=0} \Omega
$$

such that $|Y, f\rangle:=\int f(t)|Y\rangle_{t} \mathrm{~d} t$ are proper one-particle vectors. They span the one-particle space [4]. Their inner product is, in spherical coordinates,

$$
\left\langle Y, f \mid Y^{\prime}, f^{\prime}\right\rangle=(2 \pi)^{-3} \int_{0}^{\infty} \frac{p^{2} \mathrm{~d} p}{2 p} \widehat{\widehat{f}(p)} \widehat{f}^{\prime}(p) \cdot \int \mathrm{d} \sigma(\mathbf{n}) \overline{Y(p \mathbf{n})} Y^{\prime}(p \mathbf{n}),
$$

where $p=|\mathbf{p}|, \mathbf{p}=p \mathbf{n}$, and $d \sigma$ is the invariant measure on the unit sphere. The wave equation $\square \varphi=0$ states that

$$
\left|\mathbf{p}^{2} Y\right\rangle_{t}+\partial_{t}^{2}|Y\rangle_{t}=0 \Leftrightarrow\left|\mathbf{p}^{2} Y, f\right\rangle+\left|Y, \partial_{t}^{2} f\right\rangle=0
$$

i.e., these vectors have zero norm w.r.t. the inner product (2.9).

$\mathrm{SO}(3)$ acts on $Y(\mathbf{p})$ by rotation of the argument. The harmonic homogeneous polynomials $Y_{\ell}$ of degree $\ell$ carry the irreducible representation $\mathcal{D}^{(\ell)}$. Hence $\left|Y_{\ell}\right\rangle_{t}$ belong to $E_{\ell} \mathcal{H}$.

The inner product (2.9) for harmonic homogeneous polynomials is diagonal w.r.t. $\ell$, and the resulting $p$-integral in (2.9)

$$
\int_{0}^{\infty} p^{2 \ell+1} \mathrm{~d} p \overline{\widehat{f}(p)} \widehat{f}^{\prime}(p)
$$

is the two-point function of a chiral quasi-primary field ("conformal current") of scaling dimension $d=\ell+1$, which defines the inner product for the representation $U^{(\ell+1)}$ of Möb. In this way, the decomposition (2.1) for $h=0$ becomes manifest [4].

From (2.7) and the invariance of the vacuum vector $\Omega$, one reads off the action of the conformal generators on $\left|Y_{\ell}\right\rangle_{t}$ for harmonic homogeneous polynomials $Y_{\ell}$ of

\footnotetext{
3 We use the same symbol for the second-quantized generators on the Fock space, as for the generators on the one-particle space.
} 
degree $\ell$. They act (for simplicity of notation on the improper states) as differential operators on $Y$ and w.r.t. $t$ :

$$
\begin{aligned}
P_{0}\left|Y_{\ell}\right\rangle_{t} & =-i \partial_{t}\left|Y_{\ell}\right\rangle_{t}, \\
D\left|Y_{\ell}\right\rangle_{t} & =-i\left(t \partial_{t}+\ell+1\right)\left|Y_{\ell}\right\rangle_{t}, \\
K_{0}\left|Y_{\ell}\right\rangle_{t} & =-i\left(t^{2} \partial_{t}+2(\ell+1) t\left|Y_{\ell}\right\rangle_{t}\right. \\
M_{k l}\left|Y_{\ell}\right\rangle_{t} & =-i\left|\left(p_{k} \partial_{l}-p_{l} \partial_{k}\right) Y_{\ell}\right\rangle_{t} \\
P_{k}\left|Y_{\ell}\right\rangle_{t} & =\left|p_{k} Y_{\ell}\right\rangle_{t}, \\
M_{0 k}\left|Y_{\ell}\right\rangle_{t} & =t\left|p_{k} Y_{\ell}\right\rangle_{t}-\partial_{t}\left|\partial_{k} Y_{\ell}\right\rangle_{t}, \\
K_{k}\left|Y_{\ell}\right\rangle_{t} & =-t^{2}\left|p_{k} Y_{\ell}\right\rangle_{t}+2\left(t \partial_{t}+\ell\right)\left|\partial_{k} Y_{\ell}\right\rangle_{t}
\end{aligned}
$$

The equality of the Casimir operators $\frac{1}{2} M_{k l} M_{k l}$ of $\mathfrak{s o}(3)$ and $\frac{1}{2}\left(P_{0} K_{0}+K_{0} P_{0}\right)-D^{2}$ of $\mathfrak{m} \ddot{\mathfrak{o}} \mathfrak{b}$ with eigenvalues $\ell(\ell+1)$ on $\left|Y_{\ell}\right\rangle_{t}$, as well as the mass-shell condition $P_{k}^{2}=P_{0}^{2}$ can be directly verified from these formulae and (2.10).

We shall need to control the spin of the vectors on the right-hand sides. The generators of $\mathfrak{s o}(3)$ and of $\mathfrak{m o} \mathfrak{b}$ clearly commute with $E_{\ell}$, hence (2.12) and (2.13) have again spin $\ell$. The polynomial $\partial_{k} Y_{\ell}$ is again harmonic, hence $\left|\partial_{k} Y_{\ell}\right\rangle_{t}$ in (2.14) has spin $\ell-1$. Since $p_{k} Y_{\ell}-\frac{\mathbf{p}^{2}}{2 \ell+1} \partial_{k} Y_{\ell}$ is harmonic, the vector $\left|p_{k} Y_{\ell}\right\rangle_{t}$ splits into

$E_{\ell+1}\left|p_{k} Y_{\ell}\right\rangle_{t}=\left|\left(p_{k}-\frac{\mathbf{p}^{2}}{2 \ell+1} \partial_{k}\right) Y_{\ell}\right\rangle_{t}$ and $E_{\ell-1}\left|p_{k} Y_{\ell}\right\rangle_{t}=-\frac{1}{2 \ell+1} \partial_{t}^{2}\left|\partial_{k} Y_{\ell}\right\rangle_{t}$

where we have used (2.10) in the latter. All other projections vanish.

Finally, for the complex scalar field, we have two copies of states $|Y\rangle_{t}^{ \pm}$with the same actions (2.12)-(2.14) of $\mathfrak{s o}(2,4)$ and actions of the unitary charge and anti-unitary PCT operators

$$
Q|Y\rangle_{t}^{ \pm}= \pm|Y\rangle_{t}^{ \pm}, \quad J|Y\rangle_{t}^{ \pm}=|Y\rangle_{-t}^{\mp}
$$

$Q$ commutes with the conformal generators, while

$$
J P_{\mu}=P_{\mu} J, \quad J M_{\mu \nu}=-M_{\mu \nu} J, \quad J D=-D J, \quad J K_{\mu}=K_{\mu} J, \quad J Q=-Q J .
$$

\subsection{Main result}

Let $\mathcal{H}=\mathcal{H}^{+} \oplus \mathcal{H}^{-}$the one-particle space of the complex massless Klein-Gordon field, where the superscript \pm stands for the eigenvalue \pm 1 of the charge operator $Q$. 
As representations of Möb $\times \mathrm{SO}(3)$, both $\mathcal{H}^{ \pm}$decompose as

$$
\left.\mathcal{H}^{ \pm}\right|_{\mathrm{Möb} \times \mathrm{SO}(3)}=\bigoplus_{\ell=0}^{\infty} \mathcal{H}_{\ell}^{ \pm}, \quad \mathcal{H}_{\ell}^{+} \cong \mathcal{H}_{\ell}^{-} \cong U^{(\ell+1)} \otimes \mathcal{D}^{(\ell)}
$$

Let $E_{\ell}$ be the projections onto the subspaces $\mathcal{H}_{\ell}=\mathcal{H}_{\ell}^{+} \oplus \mathcal{H}_{\ell}^{-}, E^{(h)}=\sum_{\ell \geq h} E_{\ell}$, and $\mathcal{H}^{(h)}=E^{(h)} \mathcal{H}=\bigoplus_{\ell \geq h} \mathcal{H}_{\ell}$. Let $P_{\mu}, M_{\mu \nu}, D, K_{\mu}$ the generators of the conformal Lie algebra (2.4) represented on the one-particle space of the complex massless KleinGordon field.

The main result defines deformed generators $\widetilde{P}_{k}$ (the translations of the deformed QFT) in terms of the generators of the scalar QFT on the subspace $\mathcal{H}^{(h)}$ of the oneparticle space of the scalar QFT. The deformation is a simple ansatz for a vector operator of scaling dimension 1 , that (unlike the undeformed $P_{k}$ ) admits also transitions $\mathcal{H}_{\ell}^{ \pm} \rightarrow \mathcal{H}_{\ell}^{ \pm}$

Proposition 2.2 Let $h$ a non-negative integer. For $k=1,2,3$, make an ansatz for self-adjoint deformed generators $\widetilde{P}_{k}, \widetilde{M}_{0 k}, \widetilde{K}_{k}$ of $\mathfrak{m}$ on $\mathcal{H}^{(h)}=E^{(h)} \mathcal{H}$ by

$$
\begin{gathered}
\widetilde{P}_{k}:=\sum_{\ell \geq h} a_{\ell} \cdot\left(E_{\ell+1} P_{k} E_{\ell}+E_{\ell} P_{k} E_{\ell+1}\right)+\sum_{\ell \geq h} b_{\ell} \cdot Q \cdot \varepsilon_{k m n} P_{0} M_{m n} E_{\ell}, \\
2 \widetilde{M}_{0 k}:=i\left[K_{0}, \widetilde{P}_{k}\right], \quad-\widetilde{K}_{k}:=i\left[K_{0}, \widetilde{M}_{0 k}\right],
\end{gathered}
$$

where the coefficients $a_{\ell}, b_{\ell}$ are real.

(i) The deformed generators $\widetilde{P}_{k}, \widetilde{M}_{0 k}, \widetilde{K}_{k}$ satisfy the correct commutation relations (2.17) with the PCT operator.

(ii) Together with the undeformed generators $P_{0}, D, K_{0}$ of $\mathfrak{m o ̈ b}$ and $M_{k l}$ of $\mathfrak{s o}(3)$, they satisfy the conformal Lie algebra (2.4) on $\mathcal{H}^{(h)}$ if and only if

$$
a_{\ell}^{2}=\frac{(\ell+1)^{2}-h^{2}}{(\ell+1)^{2}}, \quad b_{\ell}^{2}=\frac{h^{2}}{4 \ell^{2}(\ell+1)^{2}}
$$

and all coefficients $b_{\ell}$ have the same sign.

(iii) The generators $\widetilde{P}_{k}$ as specified by (ii) satisfy the mass-shell condition on $\mathcal{H}^{(h)}$ :

$$
\sum_{k} \widetilde{P}_{k}^{2}=P_{0}^{2}
$$

(iv) The resulting representation of the Lie algebra $\mathfrak{s o}(2,4)$ integrates to a true (i.e., not a covering) representation $\widetilde{U}$ of the conformal group, which is equivalent to the irreducible representation $U_{(h)}$ of the conformal group.

Proof (i) is immediate by (2.16). To prove (ii), we start with a Lemma. 
Lemma 2.3 (i) The deformed generators (2.19), (2.20) fulfill the correct $[\mathfrak{h}, \mathfrak{m}]$ commutation relations (2.5) and (2.6) independent of the specification of the coefficients.

(ii) The remaining $[\mathfrak{m}, \mathfrak{m}]$ commutation relations are also true on $\mathcal{H}_{\ell}(\ell \geq h)$, if and only if

$$
i\left[\widetilde{P}_{k}, \widetilde{K}_{l}\right]=2 \delta_{k l} D+2 M_{k l}
$$

Proof of the Lemma (i) is obvious because the projections $E_{\ell}$ and the charge operator $Q$ commute with $\mathfrak{h}=\mathfrak{m} \ddot{o} \mathfrak{b} \oplus \mathfrak{s o}(3)$, and the remaining operators $P_{k}$ and $\varepsilon_{k m n} M_{m n}$ transform like vectors under $\mathfrak{s o}(3)$ and commute with $P_{0}$ and have the correct commutator with $D$ ["scaling dimension 1", (2.4)], while the commutators with $K_{0}$ are part of the definition.

(ii) follows by repeated application of $\operatorname{ad}_{K_{0}}$ and $\operatorname{ad}_{P_{0}}$ to (2.23), using (2.6) and (2.5).

(Alternatively, the correct Poincaré commutation relations

$$
i\left[\widetilde{P}_{k}, \widetilde{P}_{l}\right]=0, \quad i\left[\tilde{M}_{0 k}, \widetilde{P}_{l}\right]=\delta_{k l} P_{0}, \quad i\left[\tilde{M}_{0 k}, \tilde{M}_{0 l}\right]=M_{k l}
$$

would also serve the same purpose as (2.23).)

The remaining task for the proof of (ii) of the proposition is just a verification of (2.23), where $\widetilde{P}_{k}$ is given by (2.19) and

$$
\widetilde{K}_{l}=\sum_{\ell \geq h} a_{\ell} \cdot\left(E_{\ell+1} K_{k} E_{\ell}+E_{\ell} P_{k} E_{\ell+1}\right)-\sum_{\ell \geq h} b_{\ell} \cdot Q \cdot \varepsilon_{k m n} K_{0} M_{m n} E_{\ell}
$$

by (2.20). Using (2.12)-(2.14), one has to prove that for harmonic homogeneous polynomials $Y_{\ell}$ of degree $\ell$, the commutators $\left[\widetilde{P}_{k}, \widetilde{K}_{l}\right]$ make no transitions $\mathcal{H}_{\ell} \rightarrow$ $\mathcal{H}_{\ell \pm 2}$ :

$$
E_{\ell \pm 2}\left(\widetilde{P}_{k} E_{\ell \pm 1} \widetilde{K}_{l}-\widetilde{K}_{l} E_{\ell \pm 1} \widetilde{P}_{k}\right)\left|Y_{\ell}\right\rangle_{t}^{ \pm} \stackrel{!}{=} 0
$$

(which turns out to be automatically satisfied by the ansatz), as well as

$$
\begin{gathered}
E_{\ell \pm 1}\left(\widetilde{P}_{k}\left(E_{\ell \pm 1}+E_{\ell}\right) \widetilde{K}_{l}-\widetilde{K}_{l}\left(E_{\ell \pm 1}+E_{\ell}\right) \widetilde{P}_{k}\right)\left|Y_{\ell}\right\rangle_{t}^{ \pm} \stackrel{!}{=} 0, \\
E_{\ell}\left(\widetilde{P}_{k}\left(E_{\ell+1}+E_{\ell}+E_{\ell-1}\right) \widetilde{K}_{l}-\widetilde{K}_{l}\left(E_{\ell+1}+E_{\ell}+E_{\ell-1}\right) \widetilde{P}_{k}\right)\left|Y_{\ell}\right\rangle_{t}^{ \pm} \stackrel{!}{=}-2 i\left(\delta_{k l} D+M_{k l}\right)\left|Y_{\ell}\right\rangle_{t}^{ \pm},
\end{gathered}
$$

where the actions of the subsequent operators depend on the intermediate projections, given by (2.15). For example, the first term in the first condition $(2.24)($ for $\ell+1)$ becomes

$$
i a_{\ell} b_{\ell+1} Q \varepsilon_{k m n} P_{0} M_{m n} E_{\ell+1} K_{l}\left|Y_{\ell}\right\rangle_{t}^{ \pm}-i a_{\ell} b_{\ell} E_{\ell+1} P_{k} Q \varepsilon_{l m n} K_{0} M_{m n}\left|Y_{\ell}\right\rangle_{t}^{ \pm}
$$


which has to be worked out with (2.12)-(2.14). Exploiting the identity for harmonic homogeneous polynomials $Y_{\ell}$ [proven by contraction with $\varepsilon_{a k l}$ and using (2.15)]

$$
E_{\ell+1}\left|\left(\varepsilon_{l m n} p_{k}-\varepsilon_{k m n} p_{l}\right) p_{m} \partial_{n} Y_{\ell}\right\rangle_{t}=\ell \cdot \varepsilon_{j k l} E_{\ell+1}\left|p_{j} Y_{\ell}\right\rangle
$$

one finds that the first condition is satisfied if and only if

$$
\ell \cdot a_{\ell} b_{\ell}=(\ell+2) \cdot a_{\ell} b_{\ell+1} \text {. }
$$

Similarly, the second condition (2.24) reduces to

$$
\begin{aligned}
a_{\ell}^{2}+4 \ell^{2} \cdot b_{\ell}^{2} & =1, \\
a_{\ell}^{2}-a_{\ell-1}^{2} & =4(2 \ell+1) \cdot b_{\ell}^{2} .
\end{aligned}
$$

Eliminating $b_{\ell}^{2}$ from (2.26a) and (2.26b), one gets a simple recursion

$$
(\ell+1)^{2} a_{\ell}^{2}-\ell^{2} a_{\ell-1}^{2}=(\ell+1)^{2}-\ell^{2},
$$

hence $(\ell+1)^{2}\left(a_{\ell}^{2}-1\right)=$ const . The initial condition $a_{h-1}=0$ gives const $=-h^{2}$, hence (2.21). (2.25) shows that $b_{\ell}$ have constant sign.

This proves (ii). Evaluation of (2.22) on arbitrary vectors $\left|Y_{\ell}\right\rangle_{t}^{ \pm}$with the given values (2.21), yields the desired result, proving (iii).

Finally, to prove (iv) we note that the spectrum of $L_{0}$ in the deformed representation of $\mathfrak{s o}(2,4)$ is a subset of its spectrum on $\mathcal{H}^{(0)}$, hence integer. Therefore $\widetilde{U}$ integrates to a true representation of the conformal group. Knowing the multiplicities of the subrepresentations of $\left.\widetilde{U}\right|_{\text {Möb } \times \mathrm{SO}(3)}$, and comparing with (2.1), one concludes that $\widetilde{U}$ is unitarily equivalent to the irreducible representation $U_{(h)}$.

The signs of $b_{\ell}$ may be chosen positive without loss of generality, via the unitary charge conjugation. Also the coefficients $a_{\ell}$ may all be chosen positive via a unitary involution in the center of $\mathfrak{m} \ddot{\mathfrak{b}} \mathfrak{b} \oplus \mathfrak{s o}(3)$.

\subsection{Field algebras}

The construction of a local QFT on the Fock space $\mathcal{F}^{(h)}=\Gamma\left(\mathcal{H}^{(h)}\right)$ over $\mathcal{H}^{(h)}$ is routine.

The deformed representation $\widetilde{U}$ of the conformal group (equivalent to $U_{(h)}$ by Proposition 2.2(iv)) on the one-particle space $\mathcal{H}^{(h)}$ lifts to the Fock space $\mathcal{F}^{(h)}$ by second quantization.

For open intervals $I \subset \mathbb{R}$ (the time axis), let $O_{I}$ be the corresponding doublecone spanned by $I$, and define $\widetilde{A}\left(O_{I}\right):=\left.A\left(O_{I}\right)\right|_{\mathcal{F}^{(h)}}$, where $A(O)$ are the local algebras of the scalar field theory. For arbitrary doublecones, choose an interval $I$ and a conformal transformation $g$ such that $g O_{I}=O$, and define

$$
\widetilde{A}(O):=\tilde{U}(g) \widetilde{A}\left(O_{I}\right) \widetilde{U}(g)^{*} .
$$


The definition is unambiguous because if $g_{1} O_{I_{1}}=O=g_{2} O_{I_{2}}$, then $g_{2}^{-1} g_{1}\left(I_{1}\right)=$ $I_{2}$, hence $g:=g_{2}^{-1} g_{1} \in$ Möb $\times \operatorname{SO}(3)$, hence $\widetilde{U}(g) A\left(I_{1}\right) \widetilde{U}(g)^{*}=A\left(I_{2}\right)$. Thus, the net

$$
O \mapsto \widetilde{A}(O)
$$

is conformally covariant.

Because for any pair of spacelike separated doublecones, there is a conformal transformation $g$ mapping the doublecones into the forward and backward lightcones, respectively, locality follows from the Huygens property of the scalar field $\left(A\left(V_{+}\right)\right.$ commutes with $A\left(V_{-}\right)$) by covariance (see, e.g., [10]).

\subsection{Field equations}

At the level of fields, we define a symmetric and traceless field tensor $F_{j_{1} \ldots j_{h}}^{(*)}(t, \mathbf{0})$ by identification with the multiplet of derivative fields $Y_{j_{1} \ldots j_{h}}(-i \nabla) \varphi^{(*)}(t, \mathbf{0})$ (where $Y_{j_{1} \ldots j_{h}}$ is the appropriately normalized symmetric traceless tensor of harmonic polynomials of spin $\ell=h$ ) restricted to $\mathcal{F}^{(h)}$, and hence $F_{j_{1} \ldots j_{h}}^{(*)}(t, \mathbf{0}) \Omega$ with the (improper) spin- $h$ vectors $\left|Y_{j_{1} \ldots j_{h}}\right\rangle_{t}^{ \pm}$of $\mathcal{H}_{h}^{ \pm}$. We define $F_{j_{1} \ldots j_{h}}^{(*)}(t, \mathbf{x})$ by the adjoint action of $\widetilde{U}(\mathbf{x})=e^{i x_{k} \widetilde{P}_{k}}$ such that $F_{j_{1} \ldots j_{h}}^{(*)}(t, \mathbf{x}) \Omega$ are (improper) vectors in $\mathcal{H}^{(h) \pm}$. Then, $F^{(*)}(x)$ smeared within a doublecone $O$ are affiliated with the algebra $\widetilde{A}(O)$, hence they are local fields.

In order to make the identification of the deformed field thus defined with the free field of helicity $\pm h$, we have to establish the equation of motion [9]

$$
\nabla_{k} F_{k j_{2} \ldots j_{h}}=0, \quad \varepsilon_{k j m} \nabla_{k} F_{j j_{2} \ldots j_{h}}=i \partial_{t} F_{m j_{2} \ldots j_{h}}
$$

where $F=E+i B$, and $E_{j_{1} \ldots j_{h}}$ and $B_{j_{1} \ldots j_{h}}$ are symmetric traceless "electric" and "magnetic" tensors. On the time axis, we have by construction

$$
F_{j_{1} \ldots j_{h}}(t, \mathbf{0}) \Omega=\left|Y_{j_{1} \ldots j_{h}}\right\rangle_{t}^{+} \text {, hence } \nabla_{k} F_{j_{1} \ldots j_{h}}(t, \mathbf{0}) \Omega=i \widetilde{P}_{k}\left|Y_{j_{1} \ldots j_{h}}\right\rangle_{t}^{+} \text {. }
$$

With Proposition 2.2, we compute

$$
\widetilde{P}_{k}\left|Y_{j_{1} \ldots j_{h}}\right\rangle_{t}^{+}=a_{h} E_{h+1}\left|p_{k} Y_{j_{1} \ldots j_{h}}\right\rangle_{t}^{+}-2 b_{h} \cdot \sum_{a} \varepsilon_{k m n} \partial_{t}\left|p_{m} \partial_{n} Y_{j_{1} \ldots j_{h}}\right\rangle_{t}^{+}
$$

With (2.15), this implies

$$
\widetilde{P}_{k}\left|Y_{k j_{2} \ldots j_{h}}\right\rangle_{t}^{+}=0, \quad \varepsilon_{k j m} \widetilde{P}_{k}\left|Y_{j j_{2} \ldots j_{h}}\right\rangle_{t}^{+}=-\left.2 b_{h} \partial_{t}\left|\left(p_{n} \partial_{m}-p_{m} \partial_{n}\right) Y_{n j_{2} \ldots j_{h}}\right\rangle\right|^{2},
$$

because $\left(p_{k}-\frac{\mathbf{p}^{2}}{2 h+1} \partial_{k}\right) Y_{k j_{2} \ldots j_{h}}$ and $\varepsilon_{k m n} p_{m} \partial_{n} Y_{k j_{2} \ldots j_{h}}$ and $\varepsilon_{k j m}\left(p_{k}-\frac{\mathbf{p}^{2}}{2 h+1} \partial_{k}\right) Y_{j j_{2} \ldots j_{h}}$ are symmetric traceless tensors of rank $r=h-1, h-1, h$, respectively, of harmonic 
homogeneous polynomials of degree $\ell=h+1, h, h+1$, respectively, which vanish since $r \neq \ell$.

Now, the recursion $Y_{n j_{2} \ldots j_{h}}=\left(p_{n}-\frac{\mathbf{p}^{2}}{2 h-1} \partial_{n}\right) Y_{j_{2} \ldots j_{h}}$ together with harmonicity and homogeneity of $Y_{j_{2} \ldots j_{h}}$ imply $\left(p_{n} \partial_{m}-p_{m} \partial_{n}\right) Y_{n j_{2} \ldots j_{h}}=-(h+1) Y_{m j_{2} \ldots j_{h}}$. Since $2(h+1) b_{h}=1$, we conclude that the higher Maxwell equations hold on the time axis and on the vacuum vector:

$$
\nabla_{k} F_{k j_{2} \ldots j_{h}}(t, \mathbf{0}) \Omega=0, \quad \varepsilon_{k j m} \nabla_{k} F_{j j_{2} \ldots j_{h}}(t, \mathbf{0}) \Omega=i \partial_{t} F_{m j_{2} \ldots j_{h}}(t, \mathbf{0}) \Omega .
$$

The complex conjugate higher Maxwell equations for $F^{*}=E-i B$ are guaranteed by the presence of the operator $Q$ in (2.19), that switches the sign of $i$ in the right-hand side of (2.28) for the vectors $|Y\rangle_{t}^{-}$of charge -1 .

At this point, it becomes apparent how the charge of the scalar field is re-interpreted as the sign of the helicity of the higher Maxwell field.

By applying the spacelike translations $\widetilde{U}(\mathbf{x})$ to $(2.30)$, we conclude that the higher Maxwell equations on the vacuum vector hold everywhere in Minkowski space. Because $F^{(*)}$ are local fields on the time axis, by conformal covariance they are local on Minkowski spacetime. Then, the Reeh-Schlieder theorem ensures that the higher Maxwell equations hold as operator equations.

\subsection{Unitary implementation}

It is clear from our identification of the helicity-deformed representation on the subspace $E^{(h)} \mathcal{H}_{0}$ of the one-particle space for the complex scalar field with the known representation on the higher-helicity one-particle space, that there must exist a unitary operator $U$ intertwining the two representations. While our result Proposition 2.2 was found without knowing this unitary, we could identify it a posteriori for the case $h=1$ (Maxwell). We sketch the essential steps.

To prevent confusion, we rename the one-particle Hilbert space and its (improper) vectors of Sect. 2.3 as $\mathcal{H}_{\mathrm{scl}}$ and $|Y\rangle_{t}^{\mathrm{scl} \pm}=\left.Y(-i \nabla) \varphi^{(*)}(x) \Omega\right|_{x=(t, \mathbf{0})}$, and we introduce the one-particle Hilbert space of the Maxwell theory $\mathcal{H}_{\text {Maxw }}$ and its (improper) vectors

$$
|\mathbf{Y}\rangle_{t}^{\operatorname{Maxw} \pm}:=\left.\frac{1}{\sqrt{2}} \cdot Y_{j}(-i \nabla)\left(E_{j}(x) \pm i B_{j}(x)\right) \Omega\right|_{x=(t, \mathbf{0})}
$$

where $\mathbf{Y}=\left(Y_{1}, Y_{2}, Y_{3}\right)$ is a triple of polynomials, and the sum convention for vector indices is understood. We consider only the case of charge + and suppress the superscript. The case of charge - is identical, up to a change of sign, see below.

The respective inner products deriving from the respective two-point functions are

$$
\begin{aligned}
{ }_{t}^{\mathrm{scl}}\left\langle Y \mid Y^{\prime}\right\rangle_{t^{\prime}}^{\mathrm{scl}} & =(2 \pi)^{-3} \int \frac{\mathrm{d}^{3} p}{2 p^{0}} \overline{Y(\mathbf{p})} Y^{\prime}(\mathbf{p}) e^{-i\left(t-t^{\prime}\right)} \quad\left(p_{0}=|\mathbf{p}|\right), \\
\operatorname{Maxw}_{t}\left\langle\mathbf{Y} \mid \mathbf{Y}^{\prime}\right\rangle_{t^{\prime}}^{\mathrm{Maxw}} & =(2 \pi)^{-3} \int \frac{\mathrm{d}^{3} p}{2 p^{0}} \overline{Y_{i}(\mathbf{p})}\left(\delta_{i j} p_{0}^{2}-p_{i} p_{j}+i \varepsilon_{i j k} p_{0} p_{k}\right) Y_{j}^{\prime}(\mathbf{p}) e^{-i\left(t-t^{\prime}\right)} .
\end{aligned}
$$


For the conjugate field $\mathbf{E}-i \mathbf{B}$, only the $\varepsilon_{i j k}$-term would change sign. We write the latter as

$$
{ }_{t}^{\mathrm{Maxw}}\left\langle\mathbf{Y} \mid \mathbf{Y}^{\prime}\right\rangle_{t^{\prime}}^{\mathrm{Maxw}}={ }_{t}^{\mathrm{scl}}\left\langle Y_{i}\left|T_{i j}\right| Y_{j}^{\prime}\right\rangle_{t^{\prime}}^{\mathrm{scl}}
$$

where

$$
T_{i j}=\delta_{i j} \mathbf{P}_{0}^{2}-P_{i} P_{j}+i \varepsilon_{i j k} P_{0} P_{k}
$$

The null vectors of these inner products are (2.10), respectively,

$$
\left|\mathbf{p}^{2} \mathbf{Y}\right\rangle_{t}^{\text {Maxw }}+\partial_{t}^{2}|\mathbf{Y}\rangle_{t}^{\text {Maxw }}=0, \quad|\mathbf{p} Y\rangle_{t}^{\text {Maxw }}=0, \quad|\mathbf{p} \times \mathbf{Y}\rangle_{t}^{\text {Maxw }}+\partial_{t}|\mathbf{Y}\rangle_{t}^{\text {Maxw }}=0
$$

The action of the conformal generators on the vectors in $\mathcal{H}_{\text {Maxw }}$ is similar as (2.12)(2.14), with modifications due to the vector character of the field and its scaling dimension 2. They will be displayed in due context in the proof of Proposition 2.5.

We now introduce operators $V_{i}$ and $\nabla_{i}$ on $\mathcal{H}_{\mathrm{scl}}$ :

$$
V_{i}:=M_{k i} P_{k}-\frac{i}{2} P_{0} \varepsilon_{i k l} M_{k l}, \quad \nabla_{i}|Y\rangle_{t}^{\mathrm{scl}}:=\left|\partial_{i} Y\right\rangle_{t}^{\mathrm{scl}}
$$

and operators $V: \mathcal{H}_{\text {Maxw }} \rightarrow \mathcal{H}_{\text {scl }}$ and $V^{*}: \mathcal{H}_{\text {scl }} \rightarrow \mathcal{H}_{\text {Maxw }}$ :

$$
V|\mathbf{Y}\rangle_{t}^{\mathrm{Maxw}}:=V_{i}\left|Y_{i}\right\rangle_{t}^{\mathrm{scl}}, \quad V^{*}|Y\rangle_{t}^{\mathrm{scl}}:=-i|\nabla Y\rangle_{t}^{\mathrm{Maxw}}
$$

Here, $\nabla Y(\mathbf{p})$ denotes the gradient w.r.t. p. These operators are well-defined because they respect the respective null vectors.

The following Lemma and Proposition provide the desired unitary operator.

Lemma 2.4 (i) The range of $V_{i}$ is orthogonal to the $\ell=0$ subspace $E_{0} \mathcal{H}_{\mathrm{scl}}$.

(ii) It holds $T_{i j} \nabla_{j}=i V_{i}^{*}$

(iii) It holds $T_{i j}=V_{i}^{*} C^{-1} V_{j}$ where $C=\frac{1}{2} M_{k l} M_{k l}=\sum_{\ell} \ell(\ell+1) E_{\ell}$ is the Casimir operator.

(iv) $V^{*}$ is the adjoint of $V$.

Proposition 2.5 (i) It holds $V V^{*}=C$.

(ii) The operator $U=C^{-\frac{1}{2}} V: \mathcal{H}_{\text {Maxw }} \rightarrow\left(1-E_{0}\right) \mathcal{H}_{\text {scl }}$ is unitary. In other words, $U$ arises by polar decomposition of $V^{*}=U^{*} C^{\frac{1}{2}}$.

(iii) $U$ intertwines the actions of $\mathfrak{m o ̈ b} \oplus \mathfrak{s o}(3)$ on $\mathcal{H}_{\text {Maxw }}$ and $\left(1-E_{0}\right) \mathcal{H}_{\mathrm{scl}}$.

(iv) $U$ intertwines the actions of the generators $P_{k}, M_{0 k}, K_{k}$ of $\mathfrak{m}$ on $\mathcal{H}_{\mathrm{Maxw}}$ with the actions of the deformed generators of $\mathfrak{m}$ according to Proposition 2.2 on $\left(1-E_{0}\right) \mathcal{H}_{\text {scl }}$.

The proofs proceed by direct computation heavily using (2.12)-(2.14) and the present definitions. It could be rewarding to have a more elegant proof providing a better insight into the algebraic nature of the deformation. 
Proof of the Lemma (i) Obvious because $M_{k l} E_{0}=0$.

(ii) Straight computation, using (2.13).

(iii) The lengthy computation goes as follows: One first shows, for harmonic polynomials $Y_{\ell}$ of degree $\ell$, that $\nabla_{k} C^{-1} V_{j}\left|Y_{\ell}\right\rangle_{t}^{\text {scl }}=i \delta_{j k}\left|Y_{\ell}\right\rangle_{t}^{\text {scl }}+$ terms annihilated by $T_{i k}$ (summation over $k$ ), and then uses (ii). For the first step, the identities

$$
E_{\ell+1} P_{i} M_{i j} E_{\ell}=i \ell \cdot E_{\ell+1} P_{j} E_{\ell}, \quad E_{\ell+1} M_{i j} P_{i} E_{\ell}=i(\ell+2) \cdot E_{\ell+1} P_{j} E_{\ell}
$$

and their adjoint are extensively exploited.

(iv) Straight computation:

$$
\begin{aligned}
& \left.\operatorname{Maxw}_{t}\left\langle\mathbf{Y}\left|V^{*}\right| Y^{\prime}\right\rangle_{t^{\prime}}^{\mathrm{scl}} \operatorname{Def}=-i \stackrel{\operatorname{Maxw}_{t}}{\mathbf{Y}}|\nabla| \nabla Y^{\prime}\right\rangle_{t^{\prime}}^{\mathrm{Maxw}}=-i{ }_{t}^{\mathrm{scl}}\left\langle\mathbf{Y}\left|T_{i j} \nabla_{j}\right| Y^{\prime}\right\rangle_{t^{\prime}}^{\mathrm{scl}}= \\
& \text { (ii) } \left.={ }_{t}^{\mathrm{scl}}\left\langle Y_{i}\left|V_{i}^{*}\right| Y^{\prime}\right\rangle\right\rangle_{t^{\prime}}^{\mathrm{scl}}=\overline{{ }_{t^{\prime}}\left\langle Y^{\prime}\left|V_{i}\right| Y_{i}\right\rangle_{t}^{\mathrm{scl}}} \operatorname{Def}=\overline{{ }_{t}{ }^{\prime}\left\langle Y^{\prime}|V| \mathbf{Y}\right\rangle_{t}^{\mathrm{Maxw}}} .
\end{aligned}
$$

Proof of the Proposition (i) Straight computation: $V V^{*}=-i V_{i} \nabla_{i}=C$ because the $i \varepsilon_{i k l}$-terms vanish.

(ii) On the range $\left(\mathbf{1}-E_{0}\right) \mathcal{H}_{\mathrm{scl}}$ of $V, C^{-\frac{1}{2}}$ is well defined. Then, $U U^{*}=$ $C^{-\frac{1}{2}} V V^{*} C^{-\frac{1}{2}}=\mathbf{1}$ by virtue of (i). On the other hand, using (ii) of the Lemma,

$$
\left.\left.{ }_{t}^{\mathrm{Maxw}}\left\langle\mathbf{Y}\left|U^{*} U\right| \mathbf{Y}^{\prime}\right\rangle\right\rangle_{t^{\prime}}^{\mathrm{Maxw}}={ }_{t}^{\mathrm{scl}}\left\langle Y_{i}\left|V_{i}^{*} C^{-1} V_{j}\right| Y_{j}^{\prime}\right\rangle\right\rangle_{t^{\prime}}^{\mathrm{scl}}={ }_{t}^{\mathrm{scl}}\left\langle Y_{i}\left|T_{i j}\right| Y_{j}^{\prime}\right\rangle_{t^{\prime}}^{\mathrm{scl}}={ }^{\mathrm{Maxw}}\left\langle\mathbf{Y} \mid \mathbf{Y}^{\prime}\right\rangle t_{t^{\prime}}^{\mathrm{Maxw}},
$$

hence $U^{*} U=\mathbf{1}$ as well.

(iii) Because $M_{k l}$ commute with $C$ and $\mathbf{V}$ transforms as a vector, it is easily seen that the chain of maps $U^{*} P_{0} U$ takes $|\mathbf{Y}\rangle_{t}^{\text {Maxw }}$ to $-i\left|\left(p_{k} \partial_{l}-p_{l} \partial_{k}\right) \mathbf{Q}+Q_{l} \mathbf{e}_{k}-Q_{k} \mathbf{e}_{l}\right\rangle_{t}^{\text {Maxw }}$, which is the action of $M_{k l}$ in the Maxwell one-particle space; the additional terms as compared to (2.13) account for the vector character of the Maxwell field.

Because $P_{0}$ commute with $V_{i}$ and with $C, U^{*} P_{0} U$ takes $|\mathbf{Y}\rangle_{t}^{\text {Maxw }}$ to $-i \partial_{t}|\mathbf{Y}\rangle_{t}^{\mathrm{Maxw}}=$ $P_{0}\left|\mathbf{Y}_{t}\right\rangle^{\mathrm{Maxw}}$.

Because $V_{i}$ scale like $\mathbf{P}, U^{*} D U$ takes $|\mathbf{Y}\rangle_{t}^{\text {Maxw }}$ to $-i t \partial_{t}|\mathbf{Y}\rangle_{t}^{\text {Maxw }}-i \mid(\mathbf{p} \cdot \nabla) \mathbf{Y}+$ $2 \mathbf{Y}\rangle_{t}^{\mathrm{Maxw}}$, which is the action of $D$ in the Maxwell one-particle space. The factor 2 as compared to (2.12) reflects to the scaling dimension 2 of the Maxwell field. Finally, for $K_{0}$, it suffices to note that the equality of Casimir operators $\frac{1}{2} M_{k l} M_{k l}=$ $C=\frac{1}{2}\left(P_{0} K_{0}+K_{0} P_{0}\right)-D^{2}=P_{0} K_{0}-D^{2}-i D$ holds in both representations by (2.1). Since $U$ intertwines $M_{k l}, D$ and $P_{0}$, it also intertwines $C$ and $K_{0}=$ $P_{0}^{-1}\left(C+D^{2}+i D\right)$.

(iv) It suffices to verify $U P_{k} U^{*}=\widetilde{P}_{k}$. The others then follow by (2.6) because $U$ intertwines $K_{0}$. One computes the various contributions $E_{\ell^{\prime}} V P_{k} V^{*}\left|Q_{\ell}\right\rangle_{t}^{\text {scl }}$, and by dividing by the respective eigenvalues of $C^{\frac{1}{2}}$, one obtains (2.19) with the correct coefficients (2.21). 


\section{Mass deformation}

A second, and much simpler, instance of spacelike deformation is the construction of the massive Klein-Gordon field as a deformation of the spacelike translations and boosts of the massless Klein-Gordon field. It is "complementary" to the corresponding Hamiltonian deformation treated in [6].

In the present instance, we can just write down the deformed generators. Because the massive one-particle vectors have energy $p^{0} \geq m$, the spectral projection $E_{m}=$ $\theta\left(P_{0}-m\right)$ will play the role of the projection $E^{(h)}$ in Sect. 2.3.

The Lie algebra of the Poincaré group again has a symmetric space decomposition $\mathfrak{h} \oplus \mathfrak{m}$, where $\mathfrak{h}=\mathfrak{t} \oplus \mathfrak{s o}(3)$ ( $\mathfrak{t}=$ time translations), and $\mathfrak{m}$ is spanned by the spacelike momenta and the boost generators. By definition, the rotations $M_{k l}$ and the Hamiltonian $P_{0}$ remain undeformed. Thus, the deformed boosts $\widetilde{M}_{0 k}$ also determine the deformed momenta $\widetilde{P}_{k}=-i\left[P_{0}, \widetilde{M}_{0 k}\right]$.

We return to the real scalar field, and denote by $\varphi_{0}$ and $\varphi_{m}$ the massless and massive fields.

For the massless field, we write $|Y\rangle_{t}:=Y(-i \nabla) \varphi_{0}(t, \mathbf{0}) \Omega$ as before, and for the massive field denote $|Y\rangle_{t}^{m}:=Y(-i \nabla) \varphi_{m}(t, \mathbf{0}) \Omega$. The massive two-point function yields

$$
{ }^{m}\left\langle Y, f \mid Y^{\prime}, f^{\prime}\right\rangle^{m}=(2 \pi)^{-3} \int \frac{p^{2} \mathrm{~d} p}{2 p^{0}} \cdot \widehat{\widehat{f}\left(p^{0}\right)} \widehat{f}^{\prime}\left(p^{0}\right) \int \mathrm{d} \sigma(\mathbf{n}) \overline{Y(p \mathbf{n})} Y^{\prime}(p \mathbf{n}),
$$

where $p^{0}=\sqrt{p^{2}+m^{2}}, \mathbf{p}=p \mathbf{n}$, and $d \sigma$ is the invariant measure on the unit sphere. The Klein-Gordon equation becomes $\left|\mathbf{p}^{2} Y\right\rangle_{t}^{m}+\left(\partial_{t}^{2}+m^{2}\right)|Y\rangle_{t}^{m}=0$ w.r.t. the inner product (3.1).

The inner product (3.1) is diagonal w.r.t. the spin of the polynomials $Y, Y^{\prime}$. Consequently, it is a direct sum of inner products for polynomials $Y_{\ell}$ of spin $\ell$ as before

$$
{ }^{m}\left\langle Y_{\ell}, f \mid Y_{\ell}^{\prime}, f^{\prime}\right\rangle^{m}=(2 \pi)^{-3} \int \frac{p^{2 \ell+2} \mathrm{~d} p}{2 p^{0}} \cdot p^{2 \ell} \cdot \widehat{f\left(p^{0}\right)} \widehat{f}^{\prime}\left(p^{0}\right) \int \mathrm{d} \sigma(\mathbf{n}) \overline{Y_{\ell}(\mathbf{n})} Y_{\ell}^{\prime}(\mathbf{n}),
$$

Passing to the integration variable $\omega=p_{0}$, one has

$$
\frac{p^{2 \ell+2} \mathrm{~d} p}{2 p^{0}}=\frac{1}{2}\left(\omega^{2}-m^{2}\right)^{\ell+1 / 2} \mathrm{~d} \omega
$$

It will be advantageous to pass to their Fourier transforms $|Y\rangle_{\omega}^{m}=\int \mathrm{d} t e^{-i \omega t}|Y\rangle_{t}^{m}$, such that

$$
\left|\mathbf{p}^{2} Y\right\rangle_{\omega}^{m}=\left(\omega^{2}-m^{2}\right)|Y\rangle_{\omega}^{m}
$$


Then, the identification $U: \mathcal{H}_{m} \rightarrow E_{m} \mathcal{H}_{0}$ of the massive one-particle space $\mathcal{H}_{m}$ with the subspace $E_{m} \mathcal{H}_{0}$ of the massless one-particle space $\mathcal{H}_{0}$

$$
U:\left|Y_{\ell}\right\rangle_{\omega}^{m} \mapsto \sigma(\omega)^{\ell+\frac{1}{2}}\left|Y_{\ell}\right\rangle_{\omega}, \quad \text { where } \sigma(\omega)=\left(1-\frac{m^{2}}{\omega^{2}}\right)^{\frac{1}{2}},
$$

is unitary w.r.t. the respective inner products (3.1) for $m=0$ and for $m>0$. In view of (3.1), the same equation (3.4) remains true for all homogeneous polynomials of degree $\ell$; this is compatible with (2.10) and (3.3) by virtue of $\omega^{2}-m^{2}=\omega^{2} \cdot \sigma\left(\omega^{2}\right)$.

The deformed Poincaré generators on $E_{m} \mathcal{H}_{0}$ arise by the unitary conjugation $\operatorname{Ad}_{U}$ of the known action of the massive Poincaré generators on $\mathcal{H}_{m}$, i.e., the pull-back under the identification (3.4). The massive Poincaré generators $P_{\mu}$ and $M_{\mu \nu}$ act on $\left|Y_{\ell}\right\rangle_{t}^{m}$ in exactly the same way as the corresponding massless generators on $\left|Y_{\ell}\right\rangle_{t}$ in (2.12)(2.14). In particular, the deformation preserves the Hamiltonian $P_{0}$ and the generators $M_{k l}$ of rotations. The deformation of the spacelike momenta gives immediately

$$
\widetilde{P}_{k}\left|Y_{\ell}\right\rangle_{\omega}=\sigma(\omega) \cdot P_{k}\left|Y_{\ell}\right\rangle_{\omega} \Rightarrow \widetilde{P}_{k}=P_{k} \cdot\left(1-\frac{m^{2}}{P_{0}^{2}}\right)^{\frac{1}{2}} .
$$

The mass-shell condition

$$
\sum_{k} \widetilde{P}_{k}^{2}=P_{0}^{2}-m^{2}
$$

is trivially fulfilled by (3.5).

For the deformed boosts on $E_{m} \mathcal{H}_{0}$, one gets

$$
\begin{aligned}
\tilde{M}_{0 k}\left|Y_{\ell}\right\rangle_{\omega} & =\sigma(\omega)^{-\ell-\frac{1}{2}}\left(\partial_{\omega}\left(\sigma(\omega)^{\ell+\frac{3}{2}}\left|p_{k} Y_{\ell}\right\rangle_{\omega}\right)+\omega \sigma(\omega)^{\ell-\frac{1}{2}}\left|\partial_{k} Y_{\ell}\right\rangle_{\omega}\right) \\
& =\left(\left(\ell+\frac{3}{2}\right) \sigma^{\prime}(\omega)+\sigma(\omega) \partial_{\omega}\right)\left|p_{k} Y_{\ell}\right\rangle_{\omega}+\omega \sigma(\omega)^{-1}\left|\partial_{k} Y_{\ell}\right\rangle_{\omega} .
\end{aligned}
$$

Using (2.12)-(2.14), this can be seen to be equivalent to

$$
\tilde{M}_{0 k}=\left(M_{0 k}-\frac{1}{2 P_{0}}\left(D P_{k}+P_{k} D\right) \cdot \frac{m^{2}}{P_{0}^{2}}\right) \cdot\left(1-\frac{m^{2}}{P_{0}^{2}}\right)^{-\frac{1}{2}}
$$

(where the operator ordering has been adjusted so as to match the coefficient $\ell+$ $\frac{3}{2}$ ). Because in the massless one-particle representation the Casimir operators $C=$ $\frac{1}{2} M_{k l} M_{k l}$ of $\mathfrak{s o ( 3 )}$ and $\frac{1}{2}\left(P_{0} K_{0}+K_{0} P_{0}\right)-D^{2}$ of $\mathfrak{m o ̈ b}$ coincide, this can also be written

$$
\tilde{M}_{0 k}=\frac{1}{2}\left(\sigma\left(P_{0}\right) M_{0 k}+M_{0 k} \sigma\left(P_{0}\right)\right)+\frac{i}{2}\left[C, P_{k}\right] \cdot \sigma^{\prime}\left(P_{0}\right) .
$$

By construction (unitary conjugation with $U$ ) the deformed generators on the subspace $E_{m} \mathcal{H}_{0}$ are self-adjoint and satisfy the Poincaré commutation relations. Indeed, 
the hermiticity, as well as the commutator $i\left[P_{0}, \widetilde{M}_{0 k}\right]=-\widetilde{P}_{k}$, are also explicitly verified without much effort. The explicit verification of the commutation relation $i\left[\widetilde{M}_{0 k}, \widetilde{M}_{0 l}\right]=M_{k l}$ does not directly give the desired result, but rather

$$
i\left[\tilde{M}_{0 k}, \tilde{M}_{0 l}\right]=\left(M_{k l}+\left(M_{0 k} P_{l}-M_{0 l} P_{k}\right) \frac{m^{2}}{P_{0}^{3}}\right)\left(1-\frac{m^{2}}{P_{0}^{2}}\right)^{-1}
$$

This equals $M_{k l}$ on $E_{m} \mathcal{H}_{0}$ because $M_{0 k} P_{l}+M_{l 0} P_{k}+M_{k l} P_{0}=\varepsilon_{k l j} W^{j}$, and the Pauli-Lubanski operator $W^{\mu}=\frac{1}{2} \varepsilon^{\mu \nu \kappa \lambda} M_{\nu \kappa} P_{\lambda}$ vanishes in the massless scalar representation.

\section{Summary}

We have presented two families of examples of spacelike deformations that allow to construct new quantum field theories by fixing the restriction of a given QFT to the time axis, and deforming only the "transverse" symmetry generators. The remarkable feature is that the scheme admits the change of discrete quantum numbers (the helicity in our first example).

Both instances of spacelike deformation presented here make essential use of the enveloping algebra of the Lie algebra of the respective spacetime symmetry group (conformal, resp. Poincaré).

In both cases, it is true that we knew the expected deformation from the outset. But only in the mass deformation case did we know the unitary operator that transfers the massive generators to the massless one-particle space, and we used this knowledge to compute the deformed generators. In contrast, Proposition 2.2 is a uniqueness result, once the subspace is specified on which the deformation is supposed to be defined.

It is not difficult to see that one can also deform any given helicity $h^{\prime}>0$ to a helicity $h>h^{\prime}$, and any given mass $m^{\prime}>0$ to a mass $m>m^{\prime}$, as would be expected from the underlying pattern of inclusions of Hilbert spaces. One only needs to readjust the numerical coefficients of $E_{\ell^{\prime}} \widetilde{P}_{k} E_{\ell}$ in (2.19), and replace $\sigma\left(P_{0}\right)$ in (3.7) by $\sqrt{\left(P_{0}^{2}-m^{2}\right) /\left(P_{0}^{2}-m^{\prime 2}\right)}$. On the other hand, increasing mass and spin simultaneously might not be possible by lack of an inclusion of one-particle representations of the subgroup fixing the time axis. However, it seems possible to get a representation with $m>0$ and $s>0$ by deforming the direct sum of massless helicity representations running over all $|h| \leq s$, because conversely, the restriction of a massive spin- $s$ representation to the rotation subgroup is the direct sum of the restrictions of helicity$h$ representations with $|h| \leq s$.

The case of interacting theories will need methods going beyond representation theory of spacetime symmetry groups. Similar ideas leading to integrable models in two spacetime dimensions were previously pursued in [1, Sect. 4]. Here, based on an operator-algebraic deformation result for chiral conformal QFT [8], models with translation generators $P_{0}=\frac{1}{2}\left(P+m^{2} / P\right)$ and $P_{1}=\frac{1}{2}\left(P-m^{2} / P\right)$, satisfying 
the mass-shell condition $P_{0}^{2}-P_{1}^{2}=m^{2}$, were constructed starting from a Möbius covariant QFTs with generator $P$.

\section{Outlook}

Our constructions may give insights into the modular theory of local algebras for massive theories $[7,13]$, which is not as well known as for massless theories. Let us explain the idea.

In a generic QFT, if the local algebras $A\left(O_{I}\right)=A(I)$ for doublecones $O_{I}$ spanned by an interval $I$ along the time axis are given, then they are defined for general doublecones by the adjoint action of Poincare transformations. In Sects. 2.3 and 3, the deformed local algebras on the time axis arise just by restriction of the undeformed local algebras to the respective second-quantized subspace $\Gamma\left(E^{(h)}\right) \mathcal{F}_{0}$ or $\Gamma\left(E_{m}\right) \mathcal{F}_{0}$.

In the case of helicity deformations, one may adopt a different point of view, referring only to the representations of the conformal group. Namely, given a unitary representation $\widetilde{U}$ of Möb on $\mathcal{H}$ and its extension by the anti-unitary PCT operator $J$, the Brunetti-Guido-Longo construction [5] (BGL) allows to define a real Hilbert space $H\left(\mathbb{R}_{+}\right) \subset \mathcal{H}$ such that $H\left(\mathbb{R}_{+}\right) \cap i H\left(\mathbb{R}_{+}\right)=\{0\}$ and $\overline{\mathbb{C} H\left(\mathbb{R}_{+}\right)}=\mathcal{H}$. This definition uses only the dilations and $J$. Acting with $\widetilde{U}(g), g \in$ Möb, one obtains a net of real standard subspaces $I \mapsto H(I)$ on the intervals of the circle. This net of subspaces is local in the sense that the symplectic complement $H(I)^{\prime} \equiv(i H(I))^{\perp}$ of $H(I)$ coincides with $H\left(I^{\prime}\right)$, where $I^{\prime}$ is the complement of $I$ in $S^{1}$ and orthogonality $\perp$ refers to the real part of the scalar product. Upon second quantization, these properties turn into locality of a Möbius covariant chiral net of local algebras with the Reeh-Schlieder property. It trivially restricts to a net on the time axis by deleting the point $-1 \in S^{1}$ and identifying $S^{1} \backslash\{-1\}$ with $\mathbb{R}$ via the Cayley transform.

If the unitary representation of Möb extends to a representation of the fourdimensional conformal group on $\mathcal{H}$, then the net of standard subspaces on $S^{1}$ extends to a conformally covariant net $O \mapsto H(O)$ on the four-dimensional Dirac manifold, which in turn restricts to a net on Minkowski spacetime. By second quantization, one obtains a Huygens local net of local algebras $O \mapsto A(O)$. "Huygens locality" (= commutativity also at timelike distance) is a consequence of the locality along the time axis, that is guaranteed by the BGL construction.

By construction, the modular group of $H\left(\mathbb{R}_{+}\right)$is given by the dilations, and that of $H(I)$ is the one-parameter subgroup of Möb that fixes the interval $I$. It follows that the modular groups of the local algebras $A(O)$ ( $O$ a doublecone or a wedge) in the vacuum state are the subgroups of the conformal group (conjugate to boost subgroups) that fix the doublecone or wedge $O$. In the construction of Sect. 2.3, the one-particle space is given by $E^{(h)} \mathcal{H}$, the representation of the Möbius group remains undeformed, and the local subspaces and local algebras away from the time axis are constructed with the deformed translations and boosts. Because the projection $E^{(h)}$ commutes with the representation of Möb, it is automatic that the modular groups on the time axis coincide with those of the scalar field restricted to $E^{(h)} \mathcal{H}$, and away from the time axis are conjugate by deformed Poincaré transformations. 
The situation is very different in the mass deformation of Sect. 3. Because the spectral projection $E_{m}$ does not commute with the dilations, the latter are not defined on the subspace $\mathcal{H}_{m}$, and the BGL construction is not possible. Indeed, it is wellknown that in the massive case, $H_{m}\left(\mathbb{R}_{+}\right)$(to be identified with $H_{m}\left(V_{+}\right)$in the net on Minkowski spacetime) has trivial symplectic complement $([11,12])$, in contrast to the duality $\left(i H_{0}\left(\mathbb{R}_{+}\right)\right)^{\perp}=H_{0}\left(\mathbb{R}_{-}\right)$in the massless case. On the other hand, we know that the massive local subspace $H_{m}(I)$ of an interval $I$ on the time axis coincides with the local subspace $H_{m}\left(O_{I}\right)$ for the doublecone $O_{I}$ spanned by $I$; and by the work [6] of Eckmann and Fröhlich, we have a local unitary equivalence between the massive and massless time-zero algebras. Specifically, there is a unitary operator $U_{R}$ such that for intervals $I_{r}=(-r, r) \subset \mathbb{R}$ symmetric around $t=0$ and $r<R$, one has $H_{m}\left(O_{r}\right)=U_{R} H_{0}\left(O_{r}\right)$ where $O_{r}$ is the causal completion of the time-zero ball of radius $r$. Thus, the modular groups of $H_{m}\left(O_{r}\right)$ are, for $r<R$, conjugate to the known modular groups of $H_{0}\left(O_{r}\right)$ by $U_{R}$. Increasing $R$, the unitary $U_{R}$ will change, but the subspaces $H\left(I_{r}\right)$ for $r<R$ and their modular groups remain unchanged. Thus, the modular groups for $r<R_{1}<R_{2}$ commute with $U_{R_{2}} U_{R_{1}}^{*}$, and a more detailed investigation of the unitaries $U_{R}$ would be worthwhile to get a first insight into the hitherto unknown massive modular groups.

This information about the modular groups then passes to arbitary doublecones via the adjoint action of the deformed translations and boosts, as constructed in Sect. 3.

Acknowledgements Open Access funding provided by Projekt DEAL. We thank Roberto Longo for valuable discussions, and the referee for suggesting improvements. Our work was supported in part by GNAMPA-INdAM, the MIUR Excellence Department Project awarded to the Department of Mathematics, University of Rome Tor Vergata, CUP E83C18000100006, and by the ERC Advanced Grant 669240 QUEST “Quantum Algebraic Structures and Models”.

\section{Compliance with ethical standards}

Conflict of interest On behalf of all authors, the corresponding author states that there is no conflict of interest.

Open Access This article is licensed under a Creative Commons Attribution 4.0 International License, which permits use, sharing, adaptation, distribution and reproduction in any medium or format, as long as you give appropriate credit to the original author(s) and the source, provide a link to the Creative Commons licence, and indicate if changes were made. The images or other third party material in this article are included in the article's Creative Commons licence, unless indicated otherwise in a credit line to the material. If material is not included in the article's Creative Commons licence and your intended use is not permitted by statutory regulation or exceeds the permitted use, you will need to obtain permission directly from the copyright holder. To view a copy of this licence, visit http://creativecommons.org/licenses/by/4.0/.

\section{References}

1. Bischoff, M., Tanimoto, Y.: Integrable QFT and Longo-Witten endomorphisms. Ann. H. Poinc. 16, 569-608 (2015)

2. Borchers, H.-J.: Field operators as $C^{\infty}$ functions in spacelike directions. Nuovo Cim. 33, 1600-1613 (1964) 
3. Buchholz, D., Fredenhagen, K.: A C $C^{*}$-algebraic approach to interacting quantum field theories. Commun. Math. Phys. (2020). https://doi.org/10.1007/s00220-020-03700-9

4. Buchholz, D., D’Antoni, C., Longo, R.: Nuclear maps and modular structures. I. General properties. J. Funct. Anal. 88, 233-250 (1990)

5. Brunetti, R., Guido, D., Longo, R.: Modular localization and Wigner particles. Rev. Math. Phys. 14, 759-786 (2002)

6. Eckmann, J.-P., Fröhlich, J.: Unitary equivalence of local algebras in the quasi-free representation. Ann. Inst. H. Poinc. A 20, 201-209 (1974)

7. Figliolini, F., Guido, D.: The Tomita operator of the free scalar field. Ann. Inst. H. Poinc. A 51, 419-435 (1989)

8. Longo, R., Witten, E.: An algebraic construction of boundary quantum field theory. Commun. Math. Phys. 303, 213-232 (2011)

9. Longo, R., Morinelli, V., Preta, F., Rehren, K.-H.: Split property for free massless finite helicity fields. Ann. H. Poinc. 20, 2555-2584 (2019)

10. Longo, R., Morinelli, V., Rehren, K.-H.: Where infinite spin particles are localizable. Commun. Math. Phys. 345, 587-614 (2016)

11. Morinelli, V., Tanimoto, Y.: Scale and Möbius covariance in two-dimensional Haag-Kastler net. Commun. Math. Phys. 371, 619-650 (2019)

12. Sadowski, P., Woronowicz, S.L.: Total sets in quantum field theory. Rep. Math. Phys. 2, 113-120 (1971)

13. Saffary, T.: On the generator of massive modular groups. Lett. Math. Phys. 77, 235-248 (2006)

14. Summers, S.J.: A perspective on constructive quantum field theory. arXiv:1203.3991v2 (2016)

15. Weiner, M.: An algebraic version of Haag's theorem. Commun. Math. Phys. 305, 469-469 (2011)

Publisher's Note Springer Nature remains neutral with regard to jurisdictional claims in published maps and institutional affiliations. 